\title{
Systematic review and meta-analysis of randomised controlled trials of gastro-oesophageal reflux interventions for chronic cough associated with gastro-oesophageal reflux
}

\author{
A B Chang, T J Lasserson, T O Kiljander, F L Connor, J T Gaffney, L A Garske
}

\begin{abstract}
Objective To evaluate the efficacy of treatment for gastro-oesophageal reflux disease (GORD) on chronic cough in children and adults without an underlying respiratory disease.

Design Systematic review and meta-analysis.

Data sources Cochrane, Medline, and Embase databases, references from review articles.

Included studies Randomised controlled trials on GORD treatment for cough in children and adults without primary lung disease. Two reviewers independently selected studies and extracted paediatric and adult data on primary (clinical failure) and secondary outcomes.

Results 11 studies were included. Meta-analysis was limited to five studies in adults that compared proton pump inhibitors with placebo. All outcomes favoured proton pump inhibitors: the odds ratio for clinical failure (primary outcome) was 0.24 (95\% confidence interval 0.04 to 1.27 ); number needed to treat (NNT) was 5 (harm 50 to $\infty$ to benefit 2.5). For secondary outcomes, the standardised mean difference between proton pump inhibitors and placebo was $-0.51(-1.02$ to 0.01$)$ for mean cough score at the end of the trial and $-0.29(-0.62$ to $0.04)$ for change in cough score at the end of the trial. Subgroup analysis with generic inverse variance analysis showed a significant mean change in cough $(-0.41$ SD units, -0.75 to -0.07$)$.

Conclusion Use of a proton pump inhibitor to treat cough associated with GORD has some effect in some adults. The effect, however, is less universal than suggested in consensus guidelines on chronic cough and its magnitude of effect is uncertain.
\end{abstract}

\section{Introduction}

Cough is the most common symptom presenting to general practitioners. ${ }^{1}$ Chronic cough considerably impairs quality of life in adults and worries parents of children with cough. Prolonged or chronic cough has been variously defined as a cough that persists for more than three to eight weeks and non-specific cough defined as non-productive cough in the absence of identifiable respiratory disease or known cause.

Gastro-oesophageal reflux (GOR)-that is, reflux of gastric contents into the oesophagus-can be acid or non-acid. Reflux may be physiological and is associated with a range of gastrointestinal symptoms (abdominal pain, halitosis, etc) and extraoesophageal symptoms (cough, hoarseness, etc). ${ }^{3}$ Cohort studies in adults suggest that GOR disease (GORD) related to acid causes $21-41 \%$ of chronic non-specific cough. ${ }^{1}$ Guidelines on chronic cough suggest use of empirical treatment for GOR, ${ }^{45}$ including a therapeutic trial of three to six months of treatment for GORD. ${ }^{6}$ Although laboratory studies have shown a temporal relation between acid in the oesophagus and cough, some studies have shown that the cough resolves only after a mean of 169179 days after treatment. ${ }^{6}$ Other studies have shown that acid GORD is associated with, but is not the cause of, cough.

Current treatments for GORD include conservative measures (diet, positioning, etc), pharmaceuticals (acid suppressants such as histamine $\mathrm{H}_{2}$ receptor antagonists, and proton pump inhibitors; prokinetic agents such as domperidone, metoclopramide, and cisapride), and surgical approaches (fundoplication). These well established treatments for GOR, however, may not be beneficial for associated cough or may increase respiratory morbidity. ${ }^{8}$ We examined the efficacy of treatments for GOR on nonspecific chronic cough in adults and children in a systematic review. This review is based on a Cochrane systematic review. ${ }^{9}$

\section{Methods}

We used QUOROM guidelines, Cochrane collaboration method, and software (RevMan 4.2) (see bmj.com). Studies in adults and children were eligible if they were randomised controlled trials of any GORD treatment for chronic cough (lasting more than three weeks) where cough was an outcome and not primarily related to an underlying respiratory disorder. We classified the evaluated treatment regimens by type: anti-reflux conservative measures (for example, positioning, diet), $\mathrm{H}_{2}$ receptor antagonists, proton pump inhibitor, and surgical therapy. Our primary outcome was proportion of participants who were not cured at follow-up (failure to cure). Secondary outcomes were proportion of participants not substantially improved at follow-up, mean difference in cough indices (frequency of cough, scores, sensitivity), proportion who experienced adverse effects (such as rash, surgical morbidity, etc), and proportions who experienced complications (requirement for change in medication, repeat surgery, etc). We determined the proportions of participants who failed to improve on treatment using a hierarchy of assessment measures (see bmj.com).

We use the search strategy standardised by the Cochrane Airways Group as well as references in relevant publications and written communication with the authors of papers. Two reviewers independently reviewed literature searches, selected articles,

Details on the search strategy, study selection and characteristics, and data extraction and quality assessment can be found on bmj.com 


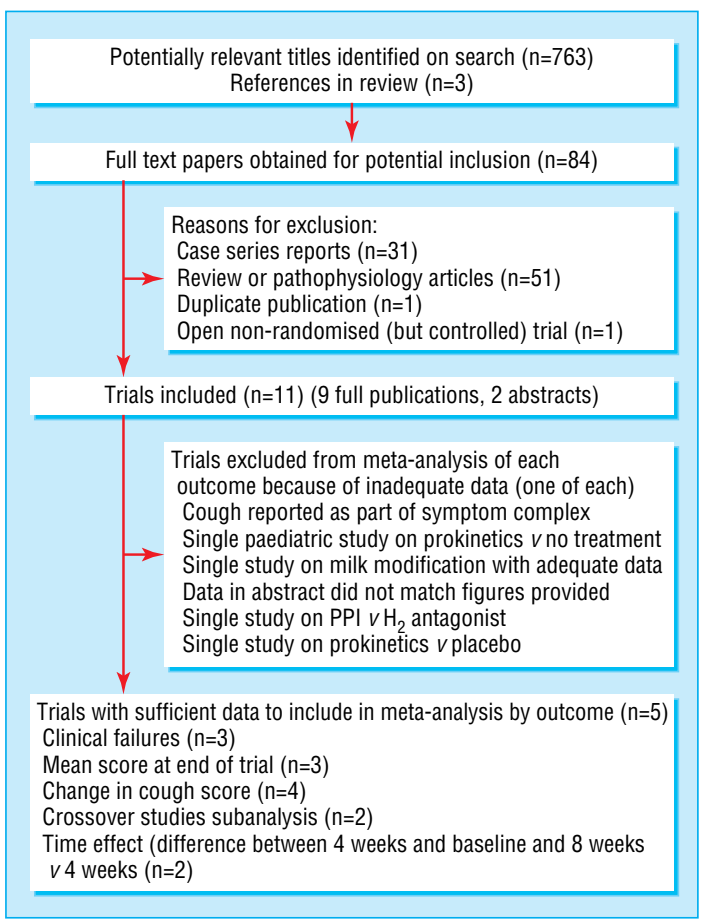

Fig 1 Details of included and excluded trials

and extracted data. We used the $\kappa$ statistic to assess agreement between reviewers. Details of other statistics including a priori, subgroup, and sensitivity analyses are on bmj.com. When we combined data with parallel studies we used only data from the first arm of crossover trials.

\section{Results}

We identified 763 potentially relevant titles and reviewed 84 papers for inclusion (fig 1). There was $92 \%$ agreement for inclusion of the 11 studies (three in children, eight in adults, $\mathrm{n}=383$ ) that met criteria for the systematic review (table). All but one ${ }^{10}$ were single centre studies; the only multicentre study was also the only study supported by industry. ${ }^{10}$ All but two studies were in English. ${ }^{11}{ }^{12}$ Additional data were sought from all authors of English articles, and two groups provided raw data. ${ }^{13}{ }^{14}$ Jadad and quality assessment scores varied (table). Agreement for quality of studies was excellent; the weighted $\kappa$ score was 0.71 for Jadad score and 0.89 for quality assessment.

\section{Paediatrics}

Two studies in children found some improvement in GORD symptoms referring to the gastrointestinal system but data for the effect on cough were inconsistent. ${ }^{10}{ }^{15}$ One study found no significant effect of cisapride or domperidone on cough associated with GORD. ${ }^{11}$ There were insufficient data in infants and children for meta-analysis and no randomised controlled trials on the use of proton pump inhibitors or surgery.

\section{Adults}

Of the eight studies in adults, six were published full articles, ${ }^{12-14} 16-18$ one was an abstract, ${ }^{19}$ and one was duplicated in a conference report and abstract. ${ }^{20}$ Five were parallel studies, and three were crossover studies. Five studies compared proton pump inhibitors with placebo but used varying doses and frequency (studies based in otolaryngology clinics ${ }^{14} 1617$ used higher doses of proton pump inhibitors, table). Although all studies included patients with cough that was presumed to be associated with GORD, criteria for entry into the studies varied. In three studies, participants enrolled through the otolaryngology department had "laryngitis" symptoms. ${ }^{14}{ }^{16}{ }^{17}$ In these studies, the exclusion criterion of primary lung disease (for example, asthma defined by hyper-responsiveness) was not as stringently applied as in the studies that enrolled participants through medical outpatients. ${ }^{12}{ }^{13} 18{ }^{20}$ In all but one study, ${ }^{12}$ the presence of GORD was confirmed objectively. No studies used non-acidic reflux as an entry criterion.

Outcome measures for all studies were various subjective cough scales; none used objective monitoring of cough. One study compared proton pump inhibitors with ranitidine ${ }^{12}$ and another was a $2 \times 2$ factorial design with cisapride and dietary intervention. ${ }^{19}$ We could not extract data from the single study on $\mathrm{H}_{2}$ receptor antagonists compared with placebo; trialists reported an improvement in cough scores with intervention in all participants and the effect was significant at two weeks. ${ }^{20}$ In the six studies that compared proton pump inhibitors or cisapride with placebo, all but one study showed no difference in improvement in cough scores in the active and placebo arms. ${ }^{12}{ }^{14-19}$ Participants took proton pump inhibitors for two to three months, and only one study followed up patients after the trial. $^{18}$

In the meta-analysis, the primary outcome (clinical failure) was the only outcome where we could use "intention to treat" data for all included studies displayed in the forest plot. The pooled odds ratio effect estimate was 0.24 (95\% confidence interval 0.04 to 1.27 (fig 2; 49 participants)). Number needed to treat for benefit was 5 (harm 50 to $\infty$ to benefit 2.5)..$^{21}$ Evaluation of the mean cough score at the end of the trial (secondary outcome), in a pooled (62 participants) analysis of three studies $^{13}{ }^{14}{ }^{16}$ showed a non-significant difference between groups (standardised mean difference $-0.51,-1.02$ to 0.01 ). When we restricted this pooled analysis to crossover studies, ${ }^{13}{ }^{14}$ the difference was also not significant $(-0.29,-0.62$ to 0.04$)$. The outcome of change in cough scores at the end of the intervention pooled from four studies (77 participants) ${ }^{13} 141617$ was not significant $(-0.29,-0.62$ to 0.04 (fig 3$)$ ). Data from the two crossover trials ${ }^{13}{ }^{14}$ that assessed mean change in symptoms showed a significant difference $(-0.41 \mathrm{SD}$ units, -0.75 to -0.07 (fig 4, 35 subjects per arm)).

Few studies determined "time to response" and duration of treatment effect was limited; after four weeks of intervention change in cough score favoured proton pump inhibitors but was not significantly different $(\mathrm{P}=0.09$, standardised mean difference $-0.51,-1.08$ to 0.06 , from two studies, ${ }^{13}{ }^{16} 51$ participants). The effect at eight weeks compared with four weeks of intervention ${ }^{13}{ }^{16}$ was also not significant $(-0.44,-1.04$ to 0.16$)$. The single study that specifically reported time to response ${ }^{18}$ was also the only study that followed up participants after the trial (open study followed by randomised controlled trial), and the effect of treatment occurred within 5-14 days. ${ }^{18}$

None of the studies reported any relevant adverse events with the interventions, and it was not possible to look for an association between level of risk and benefit. Sensitivity analyses did not alter any results.

All studies that provided sufficient data reported significant improvement in gastrointestinal symptoms. In three studies, however, there was no difference in improvement between the intervention and placebo groups. ${ }^{141617}$ All three studies on otorhinolaryngological symptoms reported significant improvement in symptoms of laryngitis over time. ${ }^{14}{ }^{17}$ In two studies, the difference in subjective and semi-objective (laryngoscopy) 
Table 1 Description of studies included in review

\begin{tabular}{|c|c|c|c|c|c|c|c|c|}
\hline Study & $\begin{array}{c}\text { Setting } \\
\text { (recruited/ } \\
\text { followed up) }\end{array}$ & $\begin{array}{l}\text { Mean age (SD or } \\
\text { range }^{\star} \text { ), sex }\end{array}$ & Methods, diagnosis & Participants & Interventions & Outcomes & Follow-up & $\begin{array}{l}\text { High quality } \\
\text { score; } \\
\text { Jadad }\end{array}$ \\
\hline \multicolumn{9}{|c|}{ Paediatric studies } \\
\hline $\begin{array}{l}\text { Dordal11 } 1994, \\
\quad \text { Spain. } \\
\text { Controlled } \\
\text { parallel } \\
\text { study }\end{array}$ & $\begin{array}{l}\text { Outpatients } \\
\text { clinic }(65 / 55)\end{array}$ & $\begin{array}{l}\text { Cisapride group: } \\
6.7 \text { years (2.97), } \\
15 \mathrm{M}: 6 \mathrm{~F} \\
\text { Domperidone } \\
\text { group: } 7.05 \text { years } \\
\text { (3.05), } 10 \mathrm{M}: 13 \mathrm{~F} \\
\text { Control: } 6.4 \text { years } \\
\text { (2.62), } 5 \mathrm{M}: 6 \mathrm{~F}\end{array}$ & $\begin{array}{l}\text { Clinical exam, skin } \\
\text { prick tests, } \\
\text { spirometry, chest x } \\
\text { ray, } \\
\text { gastro-oesophageal } \\
\text { imaging (graded } 1-4 \text {, } \\
\text { child eligible if grade } \\
\geq 2 \text { ) at baseline }\end{array}$ & $\begin{array}{l}65 \text { children (No screened not } \\
\text { given) with predominant } \\
\text { nocturnal cough (with or } \\
\text { without asthma) and GORD } \\
\text { symptoms; } 15 \text { of } 55 \text { who } \\
\text { completed trial had cough } \\
\text { without symptoms of asthma. } \\
\text { Inclusion criteria: children } \\
\text { with cough (with or without } \\
\text { co-exiting asthma), } \\
\text { predominantly nocturnal } \\
\text { symptoms, reflux symptoms. } \\
\text { Exclusions: none described }\end{array}$ & $\begin{array}{l}\text { Cisapride }(0.2 \mathrm{mg} / \mathrm{kg} 20 \\
\text { mins before each meal) with } \\
\text { domperidone }(0.2 \mathrm{mg} / \mathrm{kg} 15 \\
\text { mins before each meal) and } \\
\text { no treatment (control) for } \\
12 \text { weeks. In all: no food } \\
\text { two hours before bedtime, } \\
\text { reduce intake of acidic } \\
\text { foods, smaller more } \\
\text { frequent meals, head of bed } \\
\text { raised by } 10 \mathrm{~cm} \text {, sleeping } \\
\text { on side }\end{array}$ & $\begin{array}{l}\text { Cough; } \\
\text { gastro-imaging }\end{array}$ & $\begin{array}{l}\text { None } \\
\text { after trial } \\
\text { period }\end{array}$ & 0,1 \\
\hline $\begin{array}{l}\text { Orenstein }^{15} \\
\text { 1992, US. } \\
\text { Randomised } \\
\text { single blind, } \\
\text { crossover } \\
\text { study }\end{array}$ & $\begin{array}{l}\text { Gastro- } \\
\text { enterology clinic } \\
(25 / 25)\end{array}$ & $\begin{array}{l}\text { Median postnatal } \\
\text { age } 7.5 \text { weeks } \\
(2-26) \text {, corrected } \\
\text { age } 7 \text { weeks (-6 } \\
\text { to } 26) \text {, sex not } \\
\text { given }\end{array}$ & $\begin{array}{l}\text { Assessor blinded but } \\
\text { not care giver/nurse } \\
\text { who fed infants. } \\
\text { GORD diagnosed by } \\
\text { pHmetry, barium } \\
\text { swallow, or } \\
\text { oesophageal biopsy }\end{array}$ & $\begin{array}{l}25 \text { infants randomised (No } \\
\text { screened not given) from infants } \\
\text { referred for GORD. Inclusion } \\
\text { criteria: not defined. Exclusions: } \\
\text { concurrent acute infectious } \\
\text { respiratory illness, use of } \\
\text { prokinetic or acid reducing } \\
\text { medications }\end{array}$ & $\begin{array}{l}\text { Thickened (with rice cereal) } \\
\text { and unthickened feeds }\end{array}$ & $\begin{array}{l}\text { Cough frequency } \\
\text { during and after } \\
\text { feeds per hour }\end{array}$ & $\begin{array}{l}\text { None } \\
\text { after trial } \\
\text { period }\end{array}$ & 0,1 \\
\hline $\begin{array}{l}\text { Vanderhoof }{ }^{10} \\
\text { 2003, North } \\
\text { America. } \\
\text { Multi-centre } \\
\text { randomised } \\
\text { double blind, } \\
\text { parallel } \\
\text { study }\end{array}$ & $\begin{array}{l}\text { Six paediatric } \\
\text { centres }(110 / 98)\end{array}$ & $\begin{array}{l}\text { Intervention group: } \\
61 \text { days (4), } \\
\text { 27M:28F } \\
\text { Control group: } 58 \\
\text { days (4), 26M:23F }\end{array}$ & $\begin{array}{l}\text { Infants with } \\
\text { regurgitant reflux. } \\
\text { Parents kept diary } \\
\text { cards for two days. } \\
\text { Eligible infants } \\
\text { randomised. Infants } \\
\text { reviewed after one } \\
\text { week and } \\
\text { pharmacological } \\
\text { intervention started } \\
\text { (ranitidine after day } \\
7-8 \text { and cisapride after } \\
\text { day 14-15) }\end{array}$ & $\begin{array}{l}110 \text { infants randomised } \\
\text { (No screened not given). } \\
\text { Inclusion: } \geq 5 \text { regurgitations/day } \\
\text { for two baseline days, age } \\
14-120 \text { days, gestational age } \\
>37 \text { weeks, birth weight } \geq 2500 \\
\text { g, maternal age } \geq 18 \text { years. } \\
\text { Exclusions: congenital } \\
\text { abnormalities interfering with } \\
\text { normal feeding or causing } \\
\text { repeated regurgitation. Fever, } \\
\text { infectious illness, clinical } \\
\text { diagnosis of milk or soya } \\
\text { protein allergy, complicated } \\
\text { GORD (oesophagitis, } \\
\text { haematemesis, recurrent } \\
\text { respiratory symptoms, } \\
\text { failure to thrive), previous } \\
\text { treatment with thickened } \\
\text { formula, or treatment with } \\
\text { prokinetic medications } \\
\text { five days before start of } \\
\text { study }\end{array}$ & $\begin{array}{l}\text { Prethickened formula } \\
\text { (Enfamil AR) or placebo for } \\
35 / 36 \text { days, with equal } \\
\text { access to additional } \\
\text { pharmacological intervention }\end{array}$ & $\begin{array}{l}\text { Regurgitation } \\
\text { frequency and } \\
\text { volume, on daily } \\
\text { diary for first week } \\
\text { and then two } \\
\text { days/week. } \\
\text { Percentage feeds } \\
\text { followed by choke, } \\
\text { gag, cough. } \\
\text { Trouble sleeping. } \\
\text { Measures at week } \\
\text { one and end of } \\
\text { participation }\end{array}$ & $\begin{array}{l}\text { None } \\
\text { after trial } \\
\text { period }\end{array}$ & 2,5 \\
\hline \multicolumn{9}{|l|}{ Adult studies } \\
\hline $\begin{array}{l}\text { Eherer }^{14} 2003, \\
\text { Austria. } \\
\text { Randomised } \\
\text { double blind, } \\
\text { crossover } \\
\text { study }\end{array}$ & $\begin{array}{l}\text { Otolaryngology } \\
\text { clinic (12 with } \\
\text { cough/8) }\end{array}$ & $\begin{array}{l}48 \text { years }(20-70) \text {, } \\
16 \mathrm{M}: 5 \mathrm{~F}\end{array}$ & $\begin{array}{l}\text { Adults with } \\
\text { "laryngitis" symptoms } \\
\text { (includes cough) and } \\
\text { GORD (defined on } \\
\text { pHmetry). Video } \\
\text { laryngo-stroboscopy } \\
\text { performed and } \\
\text { screened for exclusion } \\
\text { criteria. Dual channel } \\
\text { pHmetry performed } \\
\text { and those with } \\
\text { excessive reflux } \\
\text { (>4.5\% time pH<4) } \\
\text { eligible and } \\
\text { randomised }\end{array}$ & $\begin{array}{l}21 / 22 \text { adults randomised } \\
\text { (62 screened), with GORD } \\
\text { (diagnosed on pHmetry) and } \\
\text { symptoms of laryngitis (cough, } \\
\text { nocturnal cough, sore throat, } \\
\text { hoarseness, sore dysphonic } \\
\text { attacks, globus sensation). } \\
\text { Inclusion: hoarseness for }>2 \\
\text { months, laryngitis or other } \\
\text { laryngeal symptoms which } \\
\text { includes cough. Exclusions: } \\
\text { smokers, other causes of } \\
\text { laryngitis, prior operations on } \\
\text { laryngeal area, laryngeal } \\
\text { malignancy, COPD }\end{array}$ & $\begin{array}{l}\text { Placebo or pantoprazole } 40 \\
\mathrm{mg} \text { bd for three months, } \\
\text { two weeks washout } \\
\text { followed by pantoprazole or } \\
\text { placebo for three months }\end{array}$ & $\begin{array}{l}\text { Laryngeal } \\
\text { symptoms (0-72). } \\
\text { Oesophageal } \\
\text { symptoms (0-48). } \\
\text { Laryngeal scoring. } \\
\text { Raw data given by } \\
\text { authors }\end{array}$ & $\begin{array}{l}\text { None } \\
\text { after trial } \\
\text { period }\end{array}$ & $\begin{array}{c}2,5 \\
\text { Based on } \\
\text { additional } \\
\text { information } \\
\text { given }\end{array}$ \\
\hline $\begin{array}{l}\text { Havas }^{17} 1999, \\
\text { Australia. } \\
\text { Randomised } \\
\text { double blind, } \\
\text { parallel } \\
\text { study }\end{array}$ & $\begin{array}{l}\text { Oto-laryngology } \\
\text { clinic (20/15) }\end{array}$ & $\begin{array}{l}53.6(32-76), \\
7 \mathrm{M}: 8 \mathrm{~F}\end{array}$ & $\begin{array}{l}\text { Adults with "posterior } \\
\text { pharyngolaryngitis" } \\
\text { symptoms (includes } \\
\text { cough) with or } \\
\text { without objective } \\
\text { GORD. Video } \\
\text { laryngoscopy, dual } \\
\text { channel pHmetry, } \\
\text { gastroscopy, and } \\
\text { oesophageal } \\
\text { manometry } \\
\text { performed. Distal } \\
\text { reflux defined as } \\
\text { pH }<4 \text { for }>4 \% \text { of time, } \\
\text { proximal = pH fall of } \\
\geq 3 \text { within nadir of } \leq 5 \\
\text { with oesophageal } \\
\text { acidification }\end{array}$ & $\begin{array}{l}20 \text { adults randomised from } 100 \\
\text { screened. Inclusion criteria: } \\
\text { "posterior pharyngolaryngitis" } \\
\text { assessed by video laryngoscopy } \\
\text { and symptoms (see outcome } \\
\text { measures). Exclusions: severe } \\
\text { neurological disorders, chronic } \\
\text { airflow limitation, pre-existing } \\
\text { antisecretory medications, severe } \\
\text { oesophagitis at endoscopy, } \\
\text { singers }\end{array}$ & $\begin{array}{l}\text { Lanoprazole } 30 \mathrm{mg} \text { bd or } \\
\text { placebo bd for } 12 \text { weeks }\end{array}$ & $\begin{array}{l}\text { Laryngitis } \\
\text { symptoms (cough, } \\
\text { hoarseness, throat } \\
\text { clearing, sore } \\
\text { throat). Reflux } \\
\text { symptoms } \\
\text { (dysphagia, } \\
\text { retrosternal } \\
\text { burning pain, acid } \\
\text { regurgitation, } \\
\text { odynophagia). } \\
\text { Laryngoscopy } \\
\text { findings }\end{array}$ & $\begin{array}{l}\text { None } \\
\text { after trial } \\
\text { period }\end{array}$ & 1,3 \\
\hline
\end{tabular}




\begin{tabular}{|c|c|c|c|c|c|c|c|c|}
\hline Study & $\begin{array}{l}\text { Setting } \\
\text { (recruited/ } \\
\text { followed up) }\end{array}$ & $\begin{array}{l}\text { Mean age (SD or } \\
\text { range }^{\star} \text { ), sex }\end{array}$ & Methods, diagnosis & Participants & Interventions & Outcomes & Follow-up & $\begin{array}{l}\text { High quality } \\
\text { score; } \\
\text { Jadad } \dagger\end{array}$ \\
\hline $\begin{array}{l}\text { Ing }^{20} 1997, \\
\text { Australia. } \\
\text { Double blind } \\
\text { crossover } \\
\text { study }\end{array}$ & $\begin{array}{l}\text { Medical } \\
\text { outpatients } \\
(21 / 20)\end{array}$ & $\begin{array}{l}\text { 46.8 (SEM 3.3), } \\
11 \mathrm{M}: 14 \mathrm{~F}\end{array}$ & $\begin{array}{l}\text { Chronic persistent } \\
\text { cough ( }>2 \text { months) } \\
\text { with unknown } \\
\text { aetiology after } \\
\text { standard evaluation. } \\
\text { pHmetry and } \\
\text { histamine airway } \\
\text { hyper-responsiveness. } \\
\text { pHmetry repeated in } \\
\text { seven, } 4-6 \text { weeks after } \\
\text { ranitidine treatment. } \\
\text { Significant GOR } \\
\text { defined as presence of } \\
>12 \text { reflux episodes/ } \\
24 \text { hours and time pH } \\
<4 \text { was }>0.7 \%\end{array}$ & $\begin{array}{l}25 \text { patients randomised (No } \\
\text { screened not given). All had } \\
\text { PD20 to histamine >8 } \mu \text { mols. } \\
\text { Inclusion criteria: chronic cough } \\
\text { and significant GOR. Exclusions: } \\
\text { asthma, chronic bronchitis, } \\
\text { allergic rhinitis, sinusitis, } \\
\text { postnasal drip, other respiratory } \\
\text { and cardiac diseases, acute } \\
\text { respiratory infections in } \\
\text { preceding two months, smokers, } \\
\text { or use of theophylline or ACE } \\
\text { inhibitors }\end{array}$ & $\begin{array}{l}\text { Placebo or ranitidine } 150 \\
\mathrm{mg} \text { bd for two weeks, two } \\
\text { weeks wash out followed by } \\
\text { ranitidine or placebo for two } \\
\text { weeks }\end{array}$ & $\begin{array}{l}\text { Cough scores } \\
(1-4) \text { twice daily } \\
\text { on diary cards. } \\
\text { GORD symptoms }\end{array}$ & $\begin{array}{l}\text { None } \\
\text { after trial } \\
\text { period }\end{array}$ & 1,3 \\
\hline $\begin{array}{l}\text { Jaspersen }^{12} \\
1999, \\
\text { Germany. } \\
\text { Randomised } \\
\text { parallel } \\
\text { study }\end{array}$ & $\begin{array}{l}\text { Medical } \\
\text { outpatients } \\
(20 / 20)\end{array}$ & $\begin{array}{l}\text { Omeprazole: } 49.4 \\
\text { (16.3), 5M:5F } \\
\text { Ranitidine: } 46.5 \\
\text { (19.7); 6M:5F }\end{array}$ & $\begin{array}{l}\text { Adults with chronic } \\
\text { persistent cough (>8 } \\
\text { weeks) with unknown } \\
\text { aetiology. GORD } \\
\text { symptoms or } \\
\text { gastroscopy positive }\end{array}$ & $\begin{array}{l}20 \text { adults from } 76 \text { screened, } \\
\text { with chronic cough, negative } \\
\text { clinical and radiological findings. } \\
\text { Inclusion criteria: persistent } \\
\text { cough for eight weeks, negative } \\
\text { findings from physical } \\
\text { examination, negative } \\
\text { radiological findings, with GORD } \\
\text { and normal lung function. } \\
\text { Exclusions: diagnosis of chronic } \\
\text { asthma, COPD, chronic } \\
\text { bronchitis, other oesophageal } \\
\text { disease, ACE inhibitor use, } \\
\text { ENT abnormalities, } \\
\text { smokers }\end{array}$ & $\begin{array}{l}\text { Omeprazole } 10 \mathrm{mg} / \text { day or } \\
\text { ranitidine } 300 \mathrm{mg} / \text { day for } \\
\text { eight weeks }\end{array}$ & $\begin{array}{l}\text { Cough score. } \\
\text { Oesophagitis } \\
\text { score. Outcomes } \\
\text { recorded for eight } \\
\text { weeks }\end{array}$ & $\begin{array}{l}\text { None } \\
\text { after trial } \\
\text { period }\end{array}$ & 0,1 \\
\hline $\begin{array}{l}\text { Kiljander }^{13} \\
2000, \\
\text { Finland. } \\
\text { Randomised } \\
\text { double-blind, } \\
\text { crossover } \\
\text { study }\end{array}$ & $\begin{array}{l}\text { Pulmonary } \\
\text { outpatients } \\
\text { (29/21) }\end{array}$ & $\begin{array}{l}49 \text { (IQR 20-74), } \\
\text { 10M:19F }\end{array}$ & $\begin{array}{l}\text { Adults with chronic } \\
\text { cough and GORD } \\
\text { (defined on pHmetry) } \\
\text { completed one week } \\
\text { observation by diary } \\
\text { cards, pHmetry then } \\
\text { performed and those } \\
\text { with excessive reflux } \\
\text { randomised }\end{array}$ & $\begin{array}{l}29 \text { adults randomised from } 48 \\
\text { patients screened, with GORD } \\
\text { and chronic cough ( } \geq 2 \text { months). } \\
\text { Inclusion criteria: chronic } \\
\text { persistent cough ( } \geq 2 \text { months). } \\
\text { Exclusions: abnormal chest or } \\
\text { sinus radiology, positive } \\
\text { methacholine test, rhinitis, nasal } \\
\text { mucosa appearance of } \\
\text { cobblestone or mucopurulent } \\
\text { secretions, smokers, asthma, } \\
\text { chronic bronchitis, use of ACE } \\
\text { inhibitor }\end{array}$ & $\begin{array}{l}\text { Omeprazole } 40 \mathrm{mg} \text { per day } \\
\text { or placebo for eight weeks, } \\
\text { two weeks washout } \\
\text { followed by omeprazole or } \\
\text { placebo }\end{array}$ & $\begin{array}{l}\text { Weekly daytime } \\
\text { cough and night } \\
\text { time cough. } \\
\text { Weekly gastric } \\
\text { symptoms }\end{array}$ & $\begin{array}{l}\text { None } \\
\text { after trial } \\
\text { period. } \\
\text { Raw data } \\
\text { given by } \\
\text { authors }\end{array}$ & 2,3 \\
\hline $\begin{array}{l}\text { Kopec }^{19} 2001, \\
\text { US. Partial } \\
\text { double blind } \\
\text { parallel } \\
\text { randomised } \\
\text { placebo } \\
\text { controlled } \\
\text { trial }(2 \times 2 \\
\text { factorial } \\
\text { design) }\end{array}$ & $\begin{array}{l}\text { Medical } \\
\text { outpatients } \\
(21 / 19)\end{array}$ & $53.5(12.9)$ & $\begin{array}{l}\text { Unblind randomisation } \\
\text { of diet. Double blind } \\
\text { randomisation of } \\
\text { cisapride. After } \\
\text { standard diagnostic } \\
\text { investigation for } \\
\text { chronic cough, } \\
\text { subjects who met } \\
\text { criteria (authors } \\
\text { assume that this } \\
\text { predicts patients have } \\
\text { approximate } 92 \% \\
\text { chance of having } \\
\text { chronic cough due to } \\
\text { GORD diagnosed on } \\
\text { pHmetry or on barium } \\
\text { meal) randomised }\end{array}$ & $\begin{array}{l}21 \text { adults with chronic cough } \\
\text { and GORD randomised from } \\
\text { outpatients clinic (No of eligible } \\
\text { patients not given). Inclusion } \\
\text { criteria: adults with chronic } \\
\text { cough (>8 weeks) and } \\
\text { presumably GORD related } \\
\text { cough. Exclusions: age }<18 \\
\text { years, pregnancy, known } \\
\text { contraindications to cisapride }\end{array}$ & $\begin{array}{l}2 \times 2 \text { factorial design using } \\
\text { usual care diet or antireflux } \\
\text { diet and cisapride } 10 \mathrm{mg} \\
\text { qid or placebo for four } \\
\text { months trial (four arm } \\
\text { study) }\end{array}$ & $\begin{array}{l}\text { Cough scores } \\
\text { (visual analog } \\
\text { score) }\end{array}$ & $\begin{array}{l}\text { None } \\
\text { after trial } \\
\text { period }\end{array}$ & 0,1 \\
\hline $\begin{array}{l}\text { Noordzii }^{16} \\
\text { 2001, US. } \\
\text { Randomised } \\
\text { double blind } \\
\text { parallel } \\
\text { study }\end{array}$ & $\begin{array}{l}\text { Oto-laryngology } \\
\text { clinic }(30 / 28)\end{array}$ & $\begin{array}{l}\text { Omeprazole group: } \\
51.7,8 \mathrm{M}: 7 \mathrm{~F} \\
\text { Placebo group: } \\
45.3,8 \mathrm{M}: 7 \mathrm{~F} \\
\text { (SD of age NA) }\end{array}$ & $\begin{array}{l}\text { Adults with } \\
\text { "laryngitis" symptoms } \\
\text { (includes cough) and } \\
\text { GORD (defined on } \\
\text { pHmetry). Video } \\
\text { laryngostroboscopy } \\
\text { performed and } \\
\text { screened for exclusion } \\
\text { criteria. Dual channel } \\
\text { pHmetry then } \\
\text { performed and those } \\
\text { with reflux were } \\
\text { eligible and } \\
\text { randomised. Acid } \\
\text { reflux defined as }>4 \\
\text { episodes proximal pH } \\
<4 \text { or } 3 \text { point drop in } \\
\text { pH with simultaneous } \\
\text { drop in distal pH of } \\
<4 \text { ) }\end{array}$ & $\begin{array}{l}30 \text { randomised from } 53 \text { screened } \\
\text { with GORD/laryngopharyngeal } \\
\text { reflux and symptoms of } \\
\text { laryngitis. Inclusion criteria: } \geq 1 \\
\text { symptom of laryngitis for }>3 \\
\text { months (symptoms of chronic } \\
\text { cough, hoarseness, excessive } \\
\text { phlegm, throat clearing, throat } \\
\text { pain, lump in throat and acid } \\
\text { reflux). Exclusions: viral or } \\
\text { bacterial laryngitis, benign vocal } \\
\text { fold lesions, occupational } \\
\text { exposures causing laryngitis, } \\
\text { history of seasonal allergies or } \\
\text { laryngeal malignancy }\end{array}$ & $\begin{array}{l}\text { Omeprazole } 40 \mathrm{mg} \text { bd or } \\
\text { placebo bd for two months }\end{array}$ & $\begin{array}{l}\text { Symptom scores } \\
\text { of laryngitis } \\
\text { (includes cough) } \\
\text { and GOR } \\
\text { Data on cough } \\
\text { alone provided in } \\
\text { paper }\end{array}$ & $\begin{array}{l}\text { None } \\
\text { after trial } \\
\text { period }\end{array}$ & 2,3 \\
\hline
\end{tabular}




\begin{tabular}{|c|c|c|c|c|c|c|c|c|}
\hline Study & $\begin{array}{c}\text { Setting } \\
\text { (recruited/ } \\
\text { followed up) }\end{array}$ & $\begin{array}{c}\text { Mean age (SD or } \\
\text { range }^{*} \text { ), sex }\end{array}$ & Methods, diagnosis & Participants & Interventions & Outcomes & Follow-up & $\begin{array}{l}\text { High quality } \\
\text { score; } \\
\text { Jadad† }\end{array}$ \\
\hline $\begin{array}{l}\text { Ours }^{18} 1999, \\
\text { US } \\
\text { Randomised } \\
\text { double blind } \\
\text { parallel }\end{array}$ & $\begin{array}{l}\text { Medical } \\
\text { outpatients and } \\
\text { community } \\
(17 / 17)\end{array}$ & $\begin{array}{l}\text { Age not given. } \\
\text { Median age of } 36 \\
\text { patients in study } \\
\text { before results of } \\
\text { pHmetry was } 58 \\
\text { years with cough } \\
\text { duration of five } \\
\text { years (IQR 2-10) }\end{array}$ & $\begin{array}{l}\text { Adults with chronic } \\
\text { cough and GORD } \\
\text { (defined on pHmetry). } \\
\text { Potential subjects } \\
\text { screened for exclusion } \\
\text { criteria. Patients } \\
\text { completed two week } \\
\text { observation by diary } \\
\text { cards. Those with } \\
\text { score of } \geq 3 \text { were } \\
\text { treated with } \\
\text { antihistamine and } \\
\text { nasal corticosteroids. } \\
\text { If persistent cough } \\
\text { remained (score } \geq 2 \text { ) } \\
\text { oesophageal } \\
\text { manometry and } \\
\text { pHmetry were } \\
\text { undertaken. Those } \\
\text { with GORD were } \\
\text { randomised }\end{array}$ & $\begin{array}{l}17 \text { randomised from } 71 \\
\text { screened. Patients with chronic } \\
\text { cough enrolled from newspaper } \\
\text { advertisements and outpatients } \\
\text { (most were enrolled from } \\
\text { community). Inclusion criteria: } \\
\text { aged } 18-80 \text { years with chronic } \\
\text { cough ( } \geq 6 \text { weeks) with score of } \\
\geq 2 \text { and with excessive acid } \\
\text { reflux distally or proximal. } \\
\text { Exclusions: abnormal chest } \\
\text { radiology, positive methacholine } \\
\text { test, smokers, ex-smokers } \leq 3 \\
\text { months or } \geq 20 \text { pack year history } \\
\text { of smoking, upper respiratory } \\
\text { infection within eight weeks of } \\
\text { entry, use of ACE inhibitor or } \beta \\
\text { blockers, unable to be weaned } \\
\text { off some medications, history of } \\
\text { pulmonary disease, malignancy, } \\
\text { or any comorbid condition } \\
\text { requiring treatment }\end{array}$ & $\begin{array}{l}\text { Omeprazole } 40 \mathrm{mg} \text { bd or } \\
\text { placebo for } 12 \text { weeks }\end{array}$ & $\begin{array}{l}\text { Cough severity } \\
\text { and frequency } \\
\text { scale }(0-8) \text {, day } \\
\text { and night, } \\
\text { measured daily by } \\
\text { diary cards for } 12 \\
\text { weeks. } \\
\text { Response to } \\
\text { treatment defined } \\
\text { as weekly cough } \\
\text { frequency } \\
\text { combined with } \\
\text { severity score for } \\
\text { daytime or night } \\
\text { time cough of } \leq 1 \\
\text { for } \geq 2 \text { weeks } \\
\text { consecutively }\end{array}$ & $\begin{array}{l}\text { After end } \\
\text { of trial } \\
\text { (12 } \\
\text { weeks), } \\
\text { open } \\
\text { study } \\
\text { performed } \\
\text { with } \\
\text { omeprazole } \\
\text { (one } \\
\text { month). } \\
\text { Open } \\
\text { trial } \\
\text { results } \\
\text { not used } \\
\text { for } \\
\text { analysis }\end{array}$ & 3,5 \\
\hline
\end{tabular}

$\mathrm{NA}=$ not available in publication. GORD=gastro-oesophageal reflux disease; $\mathrm{ACE}=$ angiotensin converting enzyme; $E N T=0$ torhinolaryngology; bd=twice a day; qid=four times/day. *Unless stated otherwise.

†See bmj.com for explanation of quality assessment.

improvement was similar in treatment and placebo groups. ${ }^{14} 17$ One study found significant differences between proton pump inhibitors and placebo in hoarseness and throat clearing but not in throat pain. ${ }^{16}$

\section{Discussion}

In this systematic review of the effects of treatment for GOR for cough associated with GORD, we identified 11 randomised controlled trials. In the meta-analysis of five studies, subgroup analysis indicated that treatment of cough associated with GORD improves subjective cough in participants with non-specific cough. There was no effect in the pooled analysis of the main outcomes, although all favoured proton pump inhibitors. The NNT to achieve cough resolution was 5 (the confidence interval included infinity). This review also highlights the large placebo and time period effect of treatment for chronic cough.

\section{Differences between trial types}

European $^{5}$ and American guidelines ${ }^{4}$ on the management of chronic cough recommends the use of GORD therapy (including as empirical therapy). The meta-analyses for main outcomes were not significant but all favoured treatment with proton pump inhibitors and subanalyses yielded significant results. Thus, proton pump inhibitors are probably effective in this population. Nevertheless, the small effect (considering other meta-analyses on cough $^{22}$ and large confidence intervals) of GORD treatment on cough that we found contrasts with results of non-controlled trials, which almost invariably report high rates of improvement: $100 \%$ with proton pump inhibitors alone, $86 \%$ with proton pump inhibitors with motility agents, and cure rates of $81 \%$ by surgical intervention. ${ }^{9}$ The difference between the effect of treatment of GORD on cough seen in randomised controlled trials and uncontrolled trials may be related to the period or placebo effect, which is as high as $85 \%{ }^{23}$

Other possible contributors are selection of patients, the degree of acid inhibition, length of therapy, outcomes of cough measured, role of non-acid reflux, and the presumed GORD related cough which in fact may not have been caused by GORD. Both GOR and cough are common and often coexist, but an association does not imply cause and effect. ${ }^{7}$ Our finding of an effect of treatment with proton pump inhibitors to improve cough in subgroup analysis, however, does suggest that in a proportion of adults with chronic cough and GORD, GORD is a contributing cause. The degree of acid inhibition achieved with treatment is probably a small factor, if any, given that most studies achieved a resolution of GORD gastrointestinal symptoms. It is possible, however, that different degrees of acid suppression are required to control the different manifestations of GORD. Results from the meta-analysis on length of therapy were inconclusive.

\section{Limitations of this review}

The validity of this systematic review is also hindered by the disparate nature of the interventions. In all studies participants were selected for gastrointestinal symptoms or objective evidence of

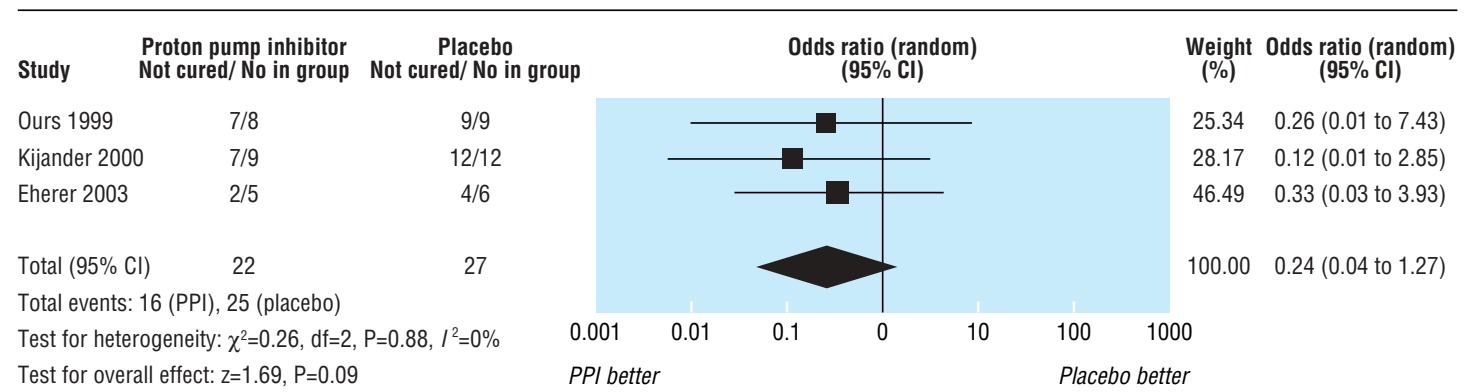

Fig 2 Meta-analysis of primary outcome (clinical failures - that is, patients still had cough at the end of the trial or reporting period), analyses by intention to treat (49 participants included in meta-analysis) 


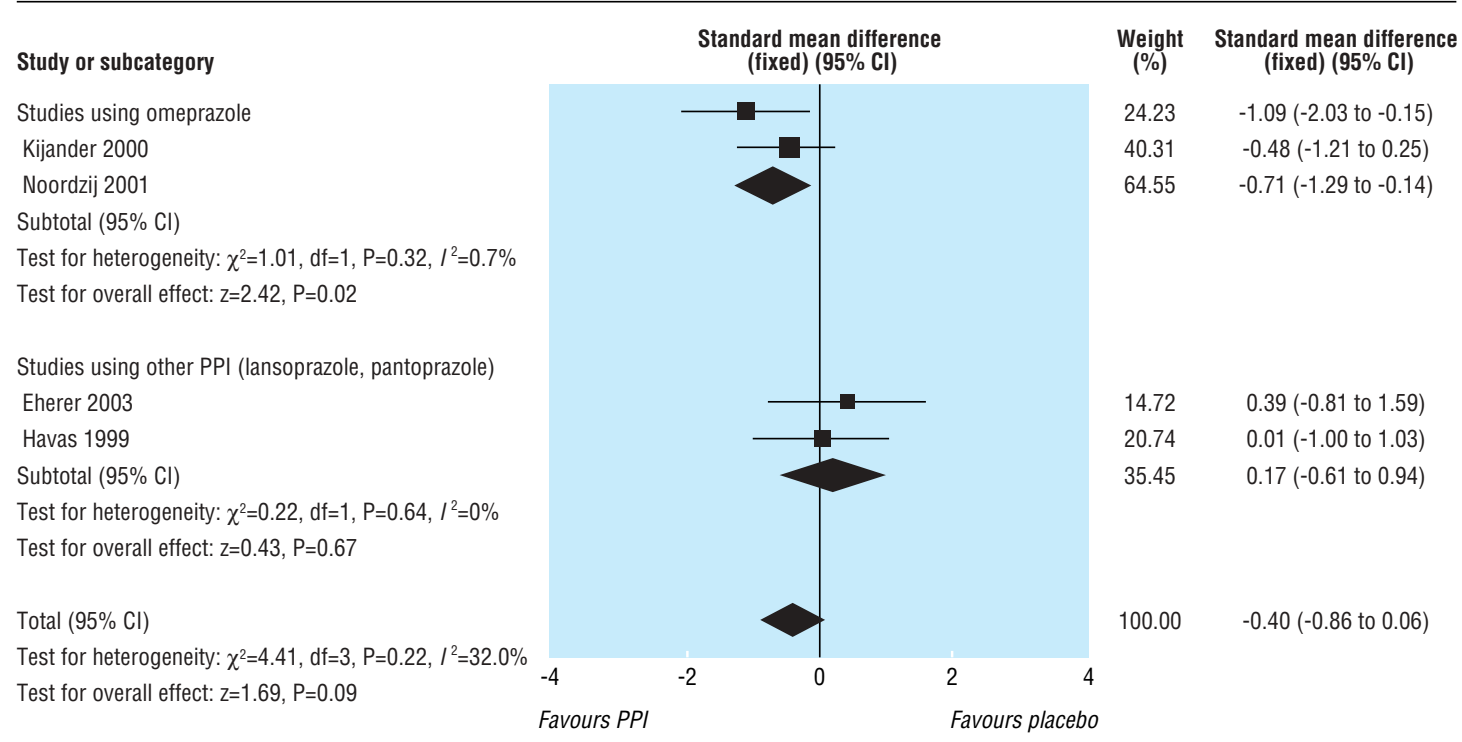

Fig 3 Meta-analysis of standardised cough scores at end of intervention (77 participants included in meta-analysis)

Study
Eherer 2003
Kijander 2000
Test for heterogeneity: $\chi^{2}=0.10, d f=1, P=0.76, I^{2}=0 \%$
Test for overall effect: $z=2.37, P=0.02$

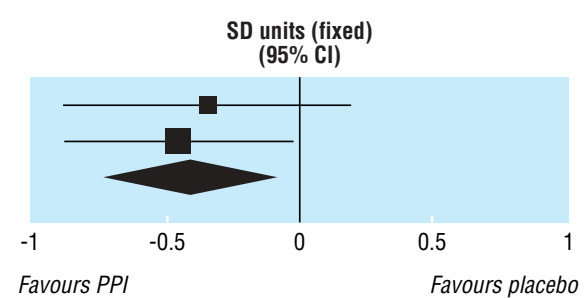

$\begin{array}{cc}\begin{array}{c}\text { Weight } \\ (\%)\end{array} & \text { SD units (fixed) } \\ (\mathbf{9 5 \%} \text { CI) }\end{array}$

Fig 4 Meta-analysis of data from the two crossover trials ${ }^{13}{ }^{14}$ assessing mean change in symptoms (subanalysis). Using generic inverse variance for crossover studies, use of proton pump inhibitors was significantly more likely than placebo to reduce cough scores at end of intervention, -0.41 SD units ( -0.75 to -0.07 )

GORD, and most, but not all, had cough. GORD criteria also varied between studies, which may have influenced results. Most studies did not use criteria for GORD specified by international guidelines. ${ }^{34}$ There are no data from randomised controlled trials on the efficacy of GORD treatment for chronic cough in the absence of GORD symptoms. In view of the possibility of period or placebo effect leading to misdiagnosis of chronic non-specific cough as due to GORD, we believe that objective confirmation of GORD is preferable to empirical therapy in patients with chronic non-specific cough and no gastrointestinal symptoms. In those with gastrointestinal symptoms, however, an empirical trial of proton pump inhibitors may be justified.

Proton pump inhibitors are currently the most potent non-surgical intervention for acid-GORD, and no adverse events were reported in these studies. The use of these drugs as a trial of therapy is probably low risk but rational prescription is required. ${ }^{8}$ Proton pump inhibitors may also cause cough and the package insert for omeprazole includes cough as an adverse event $(1.1 \%){ }^{9}$ In contrast with pharmaceutical interventions, we found no studies on surgical intervention, which is associated with a small but measurable rate of serious adverse events.

\section{Conclusion and future research}

From this systematic review and meta-analysis we conclude that proton pump inhibitors for cough associated with GORD probably have some effect in some adults, though the effect is less universal than reported in cohort studies. There are insufficient data to support or refute common recommendations to change the diet or use motility agents either as a single agent or in con- junction with proton pump inhibitors. In children, the absence of data makes specific recommendations impossible, and other causes of cough should be considered in children before any empirical treatment with a prolonged course of GORD medications or interventions. Sufficiently powered parallel placebo controlled randomised controlled trials that include an examination of "time to response," optimal duration of treatment, effects after treatment, and valid cough outcomes (objective and validated subjective scales) are required to justify international guidelines. ${ }^{45}$ Examinations of different treatments for acid and non-acid GOR are also required.

\section{What is already known on this topic}

Gastroesophageal reflux disease (GORD) is common in adults with chronic cough

International guidelines on cough recommend empirical treatment for GORD in those with chronic cough, though evidence for an effect is conflicting

\section{What this study adds}

Drugs to reduce the effect of gastric acid in GORD are beneficial in some adults with cough associated with GORD

The effect is less universal than suggested in cohort studies and international guidelines on chronic cough, and the magnitude of the clinical effect is uncertain 
We thank Chris Cates and Michael McKean for their advice, support, and comments on the protocol and review; Elizabeth Arnold for performing the relevant searches and obtaining the articles; Charlotta Pisinger and Gianni Ferrara for translations; and A J Eherer, A Ing, S E Kopec, S R Orenstein, and $\mathrm{J} A$ Vanderhoof for responding or providing additional data.

Contributors: $\mathrm{ABC}$ was responsible for initiation, design and direction of review, wrote the protocol and review, selected studies, extracted data, analysed and interpreted results, and is guarantor. LAG selected studies and extracted data, wrote the primary protocol and review, and is guarantor. TJL extracted and analysed data, interpreted results, and wrote the review. TOK wrote the review.JTG extracted data. FLC reviewed the manuscript.

Funding: $\mathrm{ABC}$ is supported by the Australian National Health and Medical Research Council and the Royal Children's Hospital Foundation. This review was supported by a bursary from the Cochrane Child Health Field. Competing interests: $\mathrm{ABC}$ has received a small grant from Glaxo for a study on asthma. TOK has received lecturing fees from AstraZeneca, Wyeth, Pfizer, and Boehringer Ingelheim, and is a consultant for AstraZeneca. LAG has received lecturing fees from AstraZeneca.

Ethical approval: Not required.

1 Morice AH. Epidemiology of cough. Pulm Pharmacol Ther 2002;15:253-9

2 Chang AB, Asher MI. A review of cough in children.J Asthma 2001;38:299-309.

3 American Gastroenterological Association medical position statement: guidelines on the use of esophageal pH recording. Gastroenterology 1996;110:1981-96.

4 Irwin RS, Boulet LP, Cloutier MM, Fuller R, Gold PM, Hoffstein V, et al. Managing cough as a defense mechanism and as a symptom. A consensus panel report of the American College of Chest Physicians. Chest 1998;114:133-81S.

5 Morice AH, Fontana GA, Sovijarvi AR, Pistolesi M, Chung KF, Widdicombe J, et al. The diagnosis and management of chronic cough. Eur Respir J 2004;24:481-92.

6 Irwin RS, Madison JM. Diagnosis and treatment of chronic cough due to gastro-esophageal reflux disease and postnasal drip syndrome. Pulm Pharmacol Ther 2002;15:261-6.

7 Laukka MA, Cameron AJ, Schei AJ. Gastroesophageal reflux and chronic cough: which comes first? J Clin Gastroenterol 1994;19:100-4.

8 Gregor JC. Acid suppression and pneumonia: a clinical indication for rational prescribing. JAMA 2004;292:2012-3.

9 Chang AB, Lasserson T, Gaffney J, Connor FC, Garske LA. Gastro-oesophageal reflux treatment for prolonged non-specific cough in children and adults. Cochrane Database Syst Rev 2005;(2):CD004823.

10 Vanderhoof JA, Moran JR, Harris CL, Merkel KL, Orenstein SR. Efficacy of a pre-thickened infant formula: a multicenter, double-blind, randomized, placebocontrolled parallel group trial in 104 infants with symptomatic gastroesophagea reflux. Clin Pediatr (Phila) 2003;42:483-95.

11 Dordal MT, Baltazar MA, Roca I, Marques L, Server MT, Botoy J. [Nocturnal spasmodic cough in the infant. Evolution after antireflux treatment.] Allerg Immunol (Paris) 1994;26:53-8.

12 Jaspersen D, Diehl KL, Geyer P, Martens E. Omeprazole in reflux-associated chronic persistent cough. Endoskopie Heute 1999;12:12-4.
13 Kiljander TO, Salomaa ER, Hietanen EK, Terho EO. Chronic cough and gastro-oesophageal reflux: a double-blind placebo-controlled study with omeprazole Eur Respir J 2000;16:633-8.

14 Eherer AJ, Habermann W, Hammer HF, Kiesler K, Friedrich G, Krejs GJ. Effect of pantoprazole on the course of reflux-associated laryngitis: a placebo-controlled double-blind crossover study. Scand J Gastroenterol 2003;38:462-7.

15 Orenstein SR, Shalaby TM, Putnam PE. Thickened feedings as a cause of increased coughing when used as therapy for gastroesophageal reflux in infants. $J$ Pediatr 1992;121:913-5

16 Noordzij JP, Khidr A, Evans BA, Desper E, Mittal RK, Reibel JF, et al. Evaluation of omeprazole in the treatment of reflux laryngitis: a prospective, placebo-controlled, randomized, double-blind study Laryngoscope 2001:111.2147-51.

17 Havas T, Huang S, Levy M, Abi-Hanna D, Truskett P, Priestly J, et al. Posterior pharyngolaryngitis: double-blind randomised placebo-controlled trial of proton pump inhibitor therapy. Austr J Otolaryngol 1999;3:243-6.

18 Ours TM, Kavuru MS, Schilz RJ, Richter JE. A prospective evaluation of esophageal testing and a double-blind, randomized study of omeprazole in a diagnostic and therapeutic algorithm for chronic cough. Am J Gastroenterol 1999;94:3131-8.

19 Kopec SE, Irwin RS, French CL, Wilson MM, Bol S. Treatment of cough due to gastroesophageal reflux disease (GERD): a double-blind randomized placebo-controlled trial comparing diet and/or cicaprode. Am J Respir Crit Care Med 2001;163(5 suppl):A64

20 Ing A. Chronic cough. Respirology 1997;2:309-16.

21 Altman DG. Confidence intervals for the number needed to treat. BMJ 1998;317:130912.

22 Marchant JM, Morris P, Gaffney J, Chang AB. Antibiotics for prolonged moist cough in children. Cochrane Database Syst Rew 2005;(4):CD004822.

23 Eccles R. The powerful placebo in cough studies? Pulm Pharmacol Ther 2002;15:303-8. 24 Rudolph CD, Mazur LJ, Liptak GS, Baker RD, Boyle JT, Colletti RB, et al. Guidelines fo evaluation and treatment of gastroesophageal reflux in infants and children: recommendations of the North American Society for Pediatric Gastroenterology and Nutrition.J Pediatr Gastroenterol Nutr 2001;32(suppl 2):S1-31.

(Accepted 27 October 2005)

doi $10.1136 /$ bmj. 38677.559005 .55

Department of Respiratory Medicine, Royal Children's Hospital, Herston, Brisbane, Old 4029, Australia

A B Chang consultant in paediatric respiratory medicine

J T Gaffney research nurse

Cochrane Airways Group, St George's Hospital Medical School, London SW17 ORE

T J Lasserson review group coordinator

Medical Center Mehiläinen, Kauppiaskatu 8, FIN-20100 Turku, Finland

$\mathrm{T} \mathrm{O}$ Kiljander consultant in respiratory medicine

Department of Gastroenterology, Royal Children's Hospital, Herston, Brisbane F L Connor consultant in paediatric gastroenterology

Princess Alexandra Hospital, Brisbane, Woolloongabba, Qld 4102, Australia L A Garske consultant in respiratory medicine

Correspondence to: A Chang annechang@ausdoctors.net 